\title{
Special Issue on Sexuality Across the Globe, Setting, Condition and Age
}

\author{
Sigmund Hough
}

Published online: 2 November 2013

(C) Springer Science+Business Media New York 2013

As we come to the close of yet another year and the beginning of a new year, a time to reflect is in order. The continuing professional challenge, excitement and wonder for what is, what is needed and what can be, motivates us forward to investigate, learn and share. The complexity of the human condition across time, age, culture, politics, and individual differences along with group similarities is the force that sustains the motivation to continue the investigation to find answers, improve healthcare and policy, and enhance quality of life. This issue brings pragmatic understanding to utilize and build upon. Still, more to do. And we look forward to a New Year!

"A journey begins with movement.

Movement continues with purpose.

Purpose develops along the journey.

Relationship begins with oneself.

Relationships begin with others.

Living successfully is individually defined.

The definition can come from within, outside, or both.

Detours, new roads, and unplanned events bring movement, excitement, challenge and relationship with oneself and with others.

Stability and prediction bring foundation.

Answers, insight and wisdom bring strength.

The opportunity to REDEFINE and RESET the journey may be the key to success and happiness. "-S.H.

Sexuality and Disability continues to provide original impact articles addressing the mental health and medical aspects of sexuality in relation to rehabilitation, hospital,

S. Hough $(\bowtie)$

396 Washington Street, Suite 211, Wellesley Hills, MA 02481, USA

e-mail: Sigmund_Hough@hms.harvard.edu 
academic and community settings, publishing up-to-date articles, case studies, clinical practice reports, reviews, featured articles, historical articles, special grand rounds topics, brief research reports and survey data reports. Value benefit is provided to authors through worldwide electronic exposure and professional access, while readership gains from scholarly contributions to advance the field through research, best-practice and educational articles. The refined lens of individual contributions from the local and international community continues to deliver a wealth of information on the topic of sexuality and disability for the reader. Thank you for being a part of our professional network.

The continued increase in our journal's 2012 Impact Factor and Citations highlights a revitalized voice between readership and authorship in the area of sexuality and disability. We want to thank our distinguished Editorial Board Members and Peer Reviewers for their work and dedication to the journal. The continued support from Springer and the wonderful talented staff highlights team commitment and high productivity in the area of sexuality and disability. 